\title{
Dosimetric advantages and clinical outcomes of simultaneous integrated boost intensity-modulated radiotherapy for anal squamous cell carcinoma
}

\author{
Katsuyuki Sakanaka, MD¹, Satoshi Itasaka, MD¹, Yuichi Ishida, MD¹, Kota Fujii, MD¹, Takahiro Horimatsu, MD², \\ Takashi Mizowaki, MD'1, Yoshiharu Sakai, MD³, Masahiro Hiraoka, MD¹ \\ Departments of 'Radiation Oncology and Image-Applied Therapy, ${ }^{2}$ Therapeutic Oncology, and \\ ${ }^{3}$ Surgery, Graduate School of Medicine, Kyoto University, Kyoto, Japan
}

Purpose: The purpose of this study was to explore the dosimetric difference between simultaneous integrated boost intensitymodulated radiotherapy (SIB-IMRT) and three-dimensional conformal radiotherapy (3DCRT), and the clinical outcomes of anal squamous cell carcinoma (ASCC) chemoradiotherapy featuring SIB-IMRT.

Materials and Methods: This study included ten patients with ASCC who underwent chemoradiotherapy using SIB-IMRT with 5-fluorouracil and mitomycin C. SIB-IMRT delivered $54 \mathrm{~Gy}$ to each primary tumor plus metastatic lymph nodes and $45 \mathrm{~Gy}$ to regional lymph nodes, in 30 fractions. Four patients received additional boosts to the primary tumors and metastatic lymph nodes; the median total dose was $54 \mathrm{~Gy}$ (range, 54 to $60 \mathrm{~Gy}$ ). We additionally created 3DCRT plans following the Radiation Therapy Oncology Group 9811 protocol to allow dosimetric comparisons with SIB-IMRT. Locoregional control, overall survival, and toxicity were calculated for the clinical outcome evaluation.

Results: Compared to 3DCRT, SIB-IMRT significantly reduced doses to the external genitalia, bladder, and intestine, delivering the doses to target and elective nodal region. At a median follow-up time of 46 months, 3 -year locoregional control and overall survival rates were $88.9 \%$ and $100 \%$, respectively. Acute toxicities were treated conservatively. All patients completed radiotherapy with brief interruptions (range, 0 to 2 days). No patient experienced $\geq$ grade 3 late toxicity during the follow-up period.

Conclusion: The dosimetric advantages of SIB-IMRT appeared to reduce the toxicity of chemoradiotherapy for ASCC achieving high locoregional control in the extended period.

Keywords: Anus neoplasms, Chemoradiotherapy, Intensity-modulated radiotherapy, Computer-assisted radiotherapy planning, Treatment outcome

\section{Introduction}

Chemoradiotherapy using three-dimensional conformal radiotherapy (3DCRT) in combination with 5 -fluorouracil plus mitomycin $\mathrm{C}$ has been the standard treatment for patients with locally advanced anal squamous cell carcinoma (ASCC). Such therapy preserves sphincter function without compromising disease control, unlike abdominal perineal

Received 21 April 2017, Revised 30 May 2017, Accepted 08 June 2017.

Correspondence: Katsuyuki Sakanaka, MD, Department of Radiation Oncology and Image-Applied Therapy, Graduate School of Medicine, Kyoto University, 54 Sho-goin Kawahara-cho, Sakyo-ku, Kyoto 606-8507, Japan. Tel: +81-75-751-3762, Fax: +81-75751-3422, E-mail: sakanaka@kuhp.kyoto-u.ac.jp

(c) This is an Open Access article distributed under the terms of the Creative Commons Attribution Non-Commercial License (http://creativecommons.org/ licenses/by-nc/4.0/) which permits unrestricted non-commercial use, distribution, and reproduction in any medium, provided the original work is properly cited.

www.e-roj.org 
resection. However, 53\%-61\% of patients experience acute non-hematological toxicities $\geq$ grade $3[1,2]$ caused by the irradiation of normal tissues surrounding the primary tumors and regional lymph nodes. Intensity-modulated radiotherapy (IMRT) is an advanced form of radiotherapy which reduces the doses to organs at risk surrounding targets. It is expected to reduce gastrointestinal and urogenital toxicities $[3,4]$.

Simultaneous integrated boost IMRT (SIB-IMRT) is one of the techniques of IMRT. It delivered different doses to the gross tumor and regional lymph nodes in a single fraction, reducing the doses to organs at risk surrounding targets. Multi-institutional phase II study (Radiation Therapy Oncology Group [RTOG] 0529) adopted SIB-IMRT technique [5], and suggested the SIB-IMRT reduced the acute toxicity than 3DCRT following the RTOG 9811 protocol. However, known to us, no studies focused on the dosimetric difference between SIBIMRT and 3DCRT. Previous planning studies of IMRT for ASCC have examined the dosimetric differences between two types of IMRT technique such as static-field IMRT vs. volumetric modulated arc therapy $[6,7]$ or between two-step IMRT vs. 3DCRT $[4,8]$.

The current study examined the dosimetric difference of SIB-IMRT over 3DCRT for ASCC, and the safety and effectiveness of chemoradiotherapy with 5-fluorouracil plus mitomycin C, when SIB-IMRT was used for ASCC.

\section{Materials and Methods}

\section{Eligibility}

Written general consent to the research use of clinical data was obtained from all patients prior to definitive chemoradiotherapy. This study (R0524) was approved by Kyoto University Institutional Review Board on April 12, 2016, and was conducted in accordance with all tenets of the Declaration of Helsinki and Japan's Ethical Guidelines for Epidemiologic Research.

The eligibility criteria were: histologically confirmed ASCC, definitive chemoradiotherapy using SIB-IMRT, the concurrent administration of continuous infusions of 5-fluorouracil and bolus infusions of mitomycin C (RTOG 9811 regimen) [1], and commencement of chemoradiotherapy from September 2011 to July 2014. In all, 10 patients met these criteria.

We collected the following information from medical records: age, sex, the Eastern Cooperative Oncology Group performance status, clinical stage, laboratory data, and radiological images including computed tomography (CT) images of the chest, abdomen, and pelvis; magnetic resonance
Table 1. Patient characteristics

\begin{tabular}{lc}
\hline \multicolumn{1}{c}{ Parameter } & Value \\
\hline Age $(\mathrm{yr})$ & $62(58-69)$ \\
Sex (female/male) & $8 / 2$ \\
Clinical stage $(\mathrm{II} / \mathrm{III} / \mathrm{IV})^{\text {a) }}$ & $6 / 3 / 1$ \\
T-status $(1 / 2 / 3 / 4)$ & $0 / 5 / 5 / 0$ \\
N-status $(0 / 1 / 2 / 3)$ & $6 / 1 / 1 / 2$ \\
M-status $(0 / 1)$ & $9 / 1^{\mathrm{b}}$ \\
\hline
\end{tabular}

Values are presented as median (interquartile range) or number.

a) Classification of clinical stage was based on the seventh edition of the TNM classification for anal cancer.

${ }^{b)}$ Ipsilateral common iliac lymph node metastases were included in the irradiation field.

images of the pelvis; and colonoscopic images. Clinical staging was defined using the TNM Classification of Malignant Tumors, 7th edition. The patient characteristics were described in Table 1.

\section{Localization and target delineation for SIB-IMRT planning}

CT simulation was performed with all patients immobilized and supine. For the accurate repositioning and intra-treatment stability, BodyFIX (Elekta AB, Stockholm, Sweden) was used. Intravenous contrast was employed to visualize the intestines and vessels. A radio-opaque marker was placed at the anal verge. The primary tumor and metastatic lymph nodes were delineated based on physical examination, radiological imaging, and colonoscopy findings.

In 10 patients treated by SIB-IMRT, the clinical target volume (CTV) for the primary tumor was created by adding $2.5 \mathrm{~cm}$ to the primary tumor. Then it was manually modified to avoid unnecessary overlap of uninvolved urogenital organs, muscles or bone, which are anatomical barriers to tumor invasion. Finally, the ventral, dorsal, and craniocaudal median margins for the primary tumor were set as $1.0 \mathrm{~cm}$ (interquartile range [IOR], 1.0 to $1.5 \mathrm{~cm})$, a $2.5 \mathrm{~cm}$, and a $2.0 \mathrm{~cm}(I Q R, 2$ to $2.5 \mathrm{~cm})$ respectively. The CTV for metastatic lymph nodes was created by adding $0.5 \mathrm{~cm}$ margins. We defined the CTV54 by combining the CTVs for the primary tumor and metastatic lymph nodes. The CTV for the elective nodal region included the CTV54 plus draining lymphatic regions, including the mesorectum, presacrum, and bilateral obturator; internal and external iliac; and inguinal nodes [9]. We defined the CTV45 by excluding the CTV54 from the CTV for the elective nodal region. We added 0.5 $\mathrm{cm}$ margins to CTV54 and the CTV for the elective nodal region to create the planning target volumes (PTV) 54 and the PTV 
for the elective nodal region. We defined PTV45 as the volume excluding PTV54 from the PTV for the elective nodal region.

We identified the external genitalia, small intestine, large intestine, bladder, femoral head, and pelvic bone as organs at risk. The intestines were delineated at least $2.0 \mathrm{~cm}$ above the most superior extent of the PTV45.

\section{Treatment planning and dose prescription of SIB-IMRT} The SIB-IMRT plans used 15-MV photon beams from a Varian CL21iX linear accelerator fitted with a Millennium 120-leaf multileaf collimator (Varian Medical Systems, Palo Alto, CA, USA). Nine static coplanar fields were used to treat 4 patients from September 2011 to May 2012. Thereafter, volumetric IMRT featuring coplanar double arcs was used to treat 6 patients (RapidArc system; Varian Medical Systems). One arc rotated clockwise from $181^{\circ}$ to $179^{\circ}$ and the other rotated counterclockwise from $179^{\circ}$ to $181^{\circ}$.

The SIB-IMRT prescribed 54 Gy to the PTV54 and 45 Gy to the PTV45 in 30 fractions. The planning goals were to create homogenous target coverage and reduce the doses to organs at risk by reference to the dose constraints of the RTOG 0529 protocol [5]. The dose calculation algorithm used was the anisotropic analytical analysis of Acuros XB, with a 2.5- $\mathrm{mm}$ grid size (Varian Medical Systems). The dose distributions included corrections for tissue heterogeneity.

\section{Dose delivery of SIB-IMRT and additional boost using 3DCRT}

SIB-IMRT was delivered 5 days per week without any planned split. Additional boosts (given via 3DCRT) were delivered to the PTV54 at the discretion of the physician, without any treatment breaks. Additional boost was attempted for the patient with cT3 and/or positive metastatic lymph nodal metastasis. Then 4 patients received boost radiotherapy after SIB-IMRT. The boost dose was $3.6 \mathrm{~Gy}$ in 2 fractions for 3 patients (T3NOMO = 1 patient, $\mathrm{T} 3 \mathrm{~N} 1 \mathrm{MO}=1$ patient, and $\mathrm{T} 3 \mathrm{~N} 3 \mathrm{MO}=1$ patient) and 6 Gy in 3 fractions for 1 patient (T2N3M1). Two patients with cT3 and/or metastatic lymph nodal metastasis didn't receive it due to severe acute toxicity. The median total dose for the 10 patients was $54 \mathrm{~Gy}(\mathrm{IOR}, 54$ to $57.6 \mathrm{~Gy})$.

\section{Details of chemoradiotherapy using SIB-IMRT}

Mitomycin $C$ was administered at a dose of $10 \mathrm{mg} / \mathrm{m}^{2}$ by bolus infusion on days 1 and 29. 5-fluorouracil was administered at a dose of $1,000 \mathrm{mg} / \mathrm{m}^{2}$ per day by continuous infusion for 24 hours on days 1-4 and 29-32 [5]. The doses of the second course of chemotherapy were reduced depending on hematological toxicities evident after the first course.

\section{Follow-up after chemoradiotherapy after SIB-IMRT}

Local responses were initially evaluated by colonoscopy up to 12 weeks after the completion of chemoradiotherapy. Then the patients were followed up via colonoscopy, digital examination, and $\mathrm{CT}$ at 4- to 12-week intervals, to check tumor progression or regression. The definition of a complete response was as follows: disappearance of the tumor, negative biopsy of the tumor bed, no mucosal erosion evident on colonoscopy, and a reduction in the shorter diameter of the metastatic lymph node to less than $5 \mathrm{~mm}$ on CT. We confirmed a complete response when these criteria were met for $>4$ weeks.

Patients were followed up every 3-6 months after confirmation of a complete response. At each follow-up, patients underwent a general physical examination and imaging. Colonoscopy and magnetic resonance imaging were used for local assessment, and CT was employed to evaluate distant metastases.

\section{3DCRT planning following RTOG 9811}

We created 3DCRT plans for all 10 patients using the same beam data in the SIB-IMRT plans. The 3DCRT plans were created by reference to the RTOG 9811 protocol [1]; the initial anterior-posterior and posterior-anterior fields included the PTV45 and PTV54 with $0.5 \mathrm{~cm}$ leaf margin except the lateral margin of the posterior-anterior field toward the inguinal nodal region of PTV45; it resulted in the inadequate dose coverage for lateral inguinal nodes, despite of sparing the bilateral femoral heads. Thus we used the supplementary anterior electron radiotherapy to cover the lateral inguinal region, which were not covered by the posterior-anterior field until $45 \mathrm{~Gy}$ in the patient with inguinal nodal metastasis. For the patients without inguinal nodal metastasis, the supplementary electron radiotherapy was used until 30.6 Gy. After the delivery of 30.6 Gy in 17 fractions, the superior fields of anterior-posterior and posterior-anterior fields were reduced to the bottom of the sacroiliac joints, and an additional $14.4 \mathrm{~Gy}$ was delivered in 8 fractions, which means both fields didn't fully cover the cranial portion of PTV45 after 30.6 Gy. Finally boost irradiation (9 Gy in 5 fractions) was delivered to the PTV54 via rotational conformal radiotherapy with $0.5 \mathrm{~cm}$ leaf margin. The gantry angles varied from $40^{\circ}$ to $140^{\circ}$ and $220^{\circ}$ to $320^{\circ}$. Doses were prescribed to the isocenter of each field. 


\section{Dosimetric comparisons between SIB-IMRT and 3DCRT plans}

To compare the target coverage of PTV54 afforded by the two techniques, we selected the maximal doses covering $2 \%$ of the structure volume $\left(D_{2 \%}\right)$, median doses $\left(D_{50 \%}\right)$, and minimum doses covering $98 \%$ of the structure volume $\left(D_{98 \%}\right)$. To evaluate the doses to organs at risk, we used structure volumes receiving $X$ Gy $\left(V_{\text {XGY }}\right)$ or higher, as follows: the external genitalia $\left(V_{206 y}, V_{306 y \text { y }}\right.$ and $\left.V_{406 y}\right)$, bladder $\left(V_{356 y,} V_{406 y}\right.$ and $\left.V_{506 y}\right)$, femoral head $\left(V_{306 y !} V_{406 y ı}\right.$ and $\left.V_{44 G y}\right)$, small and large intestines

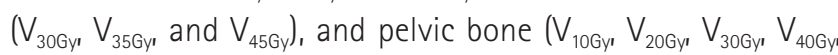
$V_{506 y}$ and a mean dose). These indices correspond to the dose constraints for organs at risk listed in RTOG 0529, except for the pelvic bone.

\section{Statistical analysis and clinical outcome}

To evaluate the dosimetric differences between the two techniques, we used the Wilcoxon signed-rank test to compare the dose indices of the PTV54, and organs at risk (the external genitalia, bladder, small and large intestines, femoral head, and pelvic bone) between 3DCRT and SIB-IMRT. Statistical analyses were conducted using GraphPad Prism version 5.03 for Windows (GraphPad Software, San Diego, CA, USA). All statistical tests were two-sided. A p-value $<0.05$ was deemed to indicate statistical significance.

We recorded the days on which radiotherapy was interrupted, overall treatment times, dosages of the second course of chemotherapy, and all acute and late adverse events, to explore the feasibility and safety of SIB-IMRT for ASCC. Adverse events that developed within 90 days after the initial day of chemoradiotherapy were considered acute adverse events. Adverse events that occurred later were considered late adverse events. All events were graded using the Common Terminology Criteria for Adverse Events v4.0.

We calculated the percentages of patients with confirmed complete responses. The times to such confirmation from the first day of chemoradiotherapy were measured and recurrence patterns were evaluated. The locoregional control (LRC), progression-free survival (PFS), and overall survival (OS) were calculated using the Kaplan-Meier method. LRC events were locoregional progression; patients were censored on the dates that locoregional control was most recently confirmed. PFS events were recurrence or death from any cause, and patients were censored on the date on which progression-free status was most recently confirmed. OS events were death from any cause, and patients were censored at their final follow-ups.

\section{Results}

\section{Dose volume indices and dose distributions of SIB- IMRT and 3DCRT}

In SIB-IMRT plans, $\mathrm{D}_{2 \%,} \mathrm{D}_{50 \%}$ and $\mathrm{D}_{98 \%}$ of PTVs were $105.6 \%$ (IOR, 104.3\% to 108.4\%), $102.0 \%$ (IOR, $101.2 \%$ to $102.6 \%)$ and 94.1\% (IQR, 90.1\% to 95.7\%) in PTV54, and 95.6\% (IQR, 94.7\% to $97.6 \%), 88.0 \%$ (IOR, $87.0 \%$ to $89.4 \%), 80.9 \%$ (IQR, $78.8 \%$ to 82.2\%) in PTV45, respectively (Reference dose was 54 Gy). $D_{50 \%}$ of CTV54 and CTV45 were 102.5\% (IOR, 102.0\% to 103.6\%) and $89.4 \%$ (IOR, 87.8\% to 91.0\%) (Reference dose was 54 Gy). Then SIB-IMRT reduced the doses to the intestine, bladder, and external genitalia, compared to 3DCRT plans (Figs. 1, 2 and Table 2). No significant difference in femoral head dosing was evident between the two techniques. The volume of the pelvic bone receiving 10-40 Gy was significantly higher on SIB-IMRT than 3DCRT (Table 2, Fig. 2).

\section{Feasibility and acute toxicities}

Two patients required radiotherapy interruptions because of hematological adverse events developing after the first course of chemotherapy. The interruption periods were 1 and 2 days. The median interruption interval for the 10 patients was 0 days. Radiotherapy was never interrupted by a nonhematological adverse event. The overall treatment time (including that of boost radiotherapy) for the 10 patients was 45 days (IQR, 43 to 45 days).

Grade 4 acute hematological and $\geq$ grade 3 acute nonhematological adverse events occurred in five and three patients, respectively (Table 3 ). The doses for the second course of chemotherapy were reduced in 5 patients; 4 received $50 \%-80 \%$ of the doses of the first course and 1 received 5-fluorouracil alone.

\section{LRC, PFS, OS and recurrence}

All patients were alive at the last follow-up. The median follow-up period was 46 months (IQR, 40 to 54 months). Complete responses were confirmed in all patients. The median time to such confirmation was 8.5 months (IQR, 6 to 12 months). Two patients experienced recurrence after confirmation of a complete response. One patient in clinical stage IIIB (T3N3M0) developed local intraluminal recurrence 32 months after the initial day of chemoradiotherapy (Fig. 3). She received palliative chemotherapy because she refused to receive the salvage abdominal-perineal resection. Another patient in clinical stage IV (T2N2M1) experienced left femoral lymph node recurrence, located distally in the initially 
A
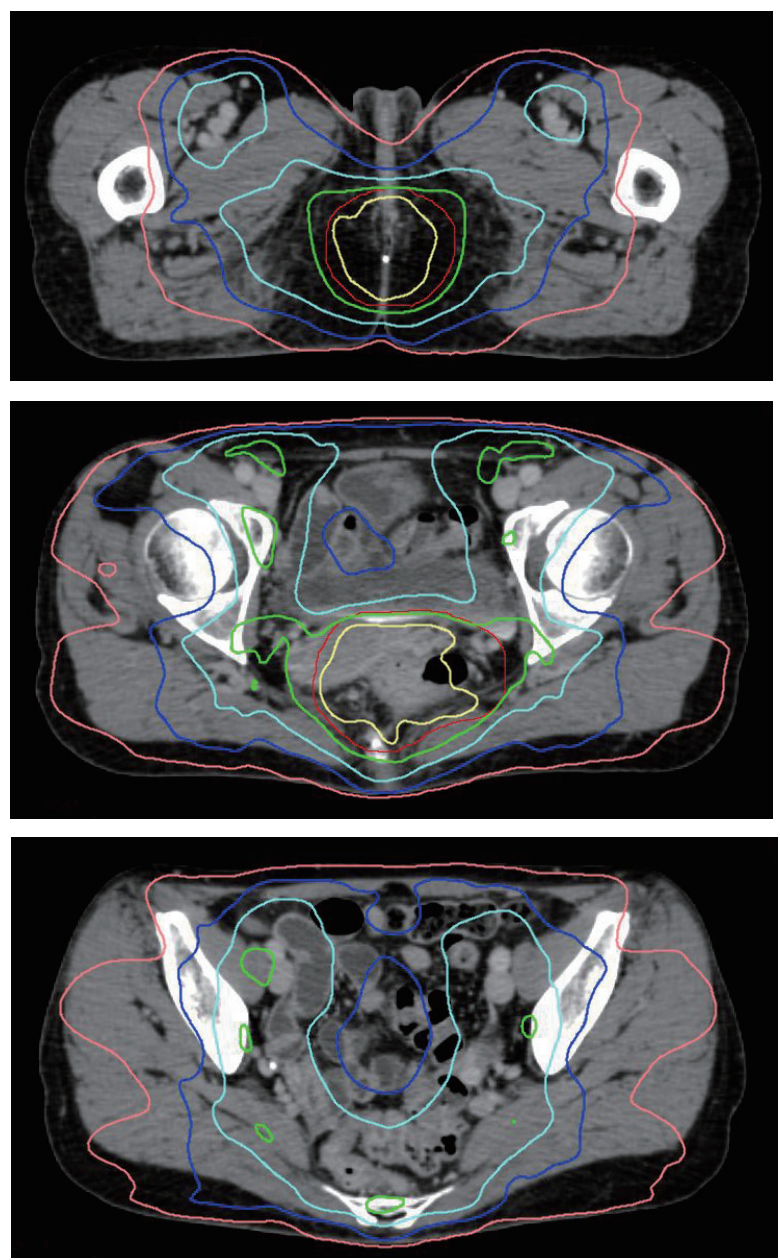

5940

5400

4860

4000

3000

2000

(cosy)

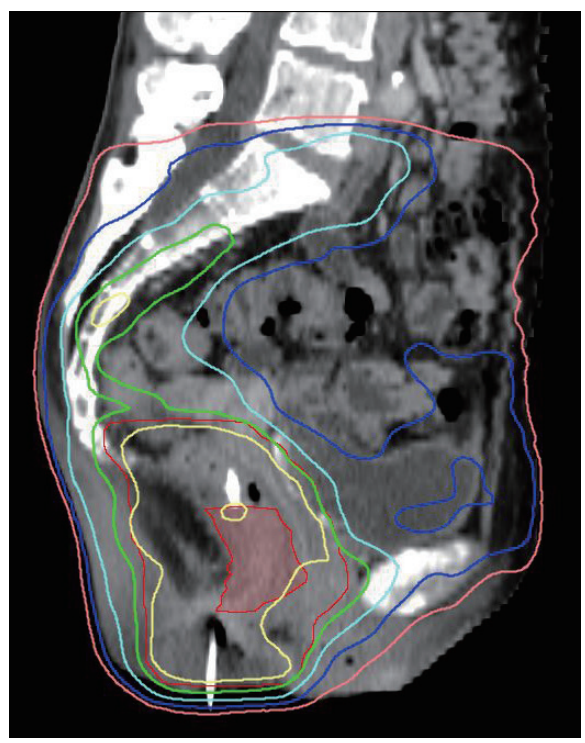

B
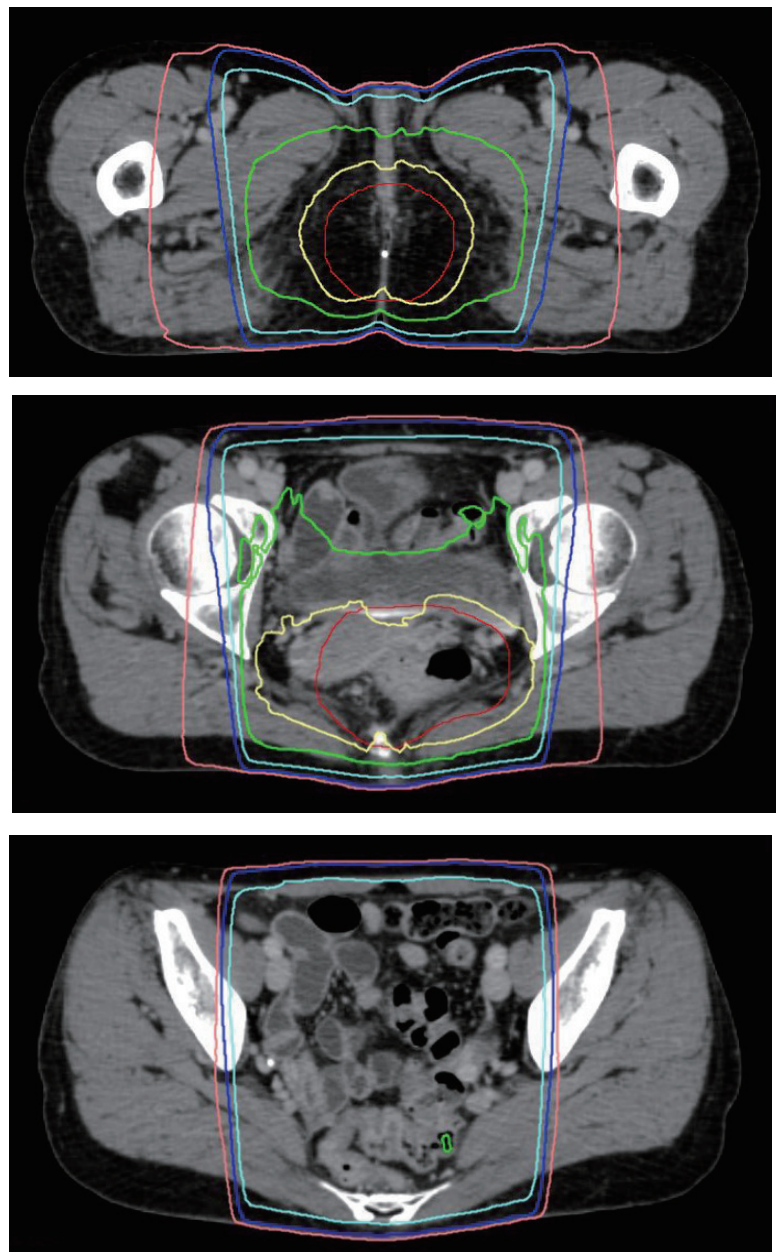

5940

5400

4860

4000

3000

2000

(coy)

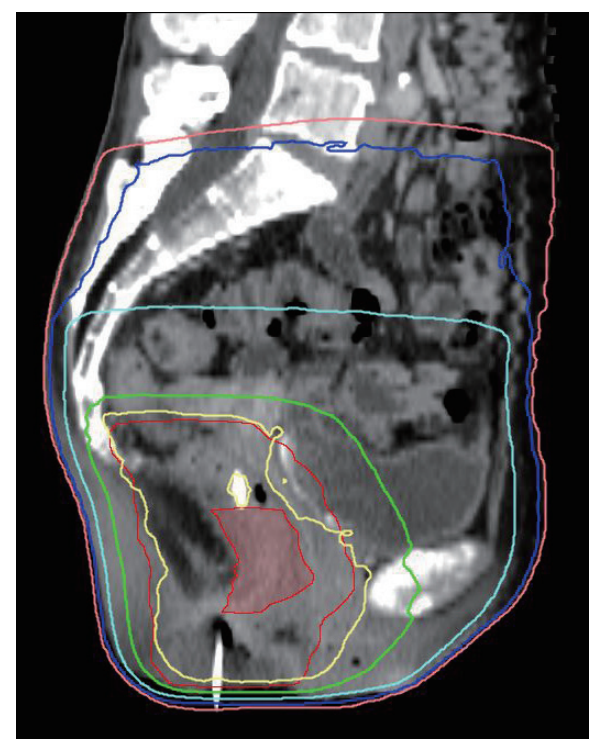

Fig. 1. Multi-plane slices of the dose distribution of (A) simultaneous integrated boost intensity-modulated radiotherapy and (B) threedimensional conformal radiotherapy. Red translucent contour = primary tumor; red contour = planning target volume for primary tumor. 
Table 2. Dose-volume indices of targets and organs at risk

\begin{tabular}{|c|c|c|c|}
\hline Dose index & 3DCRT & IMRT & $p$-value \\
\hline \multicolumn{4}{|l|}{ PTV54 } \\
\hline Volume (mL) & \multicolumn{2}{|c|}{$558.7(404.3-693.1)$} & \\
\hline$D_{2 \%}(\%)^{a)}$ & $106.6(106.2-108.3)$ & $105.6(104.3-108.4)$ & 0.3223 \\
\hline$D_{50 \%}(\%)^{a)}$ & $102.9(102.8-104.7)$ & $102.0(101.2-102.6)$ & 0.0645 \\
\hline$D_{98 \%}(\%)^{a)}$ & 99.3 (99.0-99.9) & $94.1(90.1-95.7)$ & 0.002 \\
\hline \multicolumn{4}{|l|}{ External genitalia } \\
\hline Volume (mL) & \multicolumn{2}{|c|}{$53.7(48.1-85.2)$} & \\
\hline$V_{20 G y}(\%)$ & 99.6 (93.5-99.8) & $59.1(50.3-63.7)$ & 0.0039 \\
\hline $\mathrm{V}_{30 \mathrm{~Gy}}(\%)$ & 95.3 (82.1-96.9) & $11.8(5.7-20.7)$ & 0.002 \\
\hline$V_{40 G y}(\%)$ & 74.8 (70.5-82.9) & $1.5(0.1-5.9)$ & 0.002 \\
\hline \multicolumn{4}{|l|}{ Bladder } \\
\hline Volume (mL) & \multicolumn{2}{|c|}{$101(72.6-136.8)$} & \\
\hline $\mathrm{V}_{35 \mathrm{~Gy}}(\%)$ & $100(100-100)$ & $67.3(55.6-77.9)$ & 0.0039 \\
\hline $\mathrm{V}_{40 \mathrm{~Gy}}(\%)$ & $100(100-100)$ & $51.3(36.5-64.7)$ & 0.002 \\
\hline$V_{50 G y}(\%)$ & $63.3(49.8-88.2)$ & $9.6(3.1-15.7)$ & 0.0039 \\
\hline \multicolumn{4}{|l|}{ Small intestine } \\
\hline $\mathrm{V}_{30 \mathrm{~Gy}}(\mathrm{~mL})$ & \multicolumn{2}{|c|}{$214.3(123.5-329.6)$} & 0.0195 \\
\hline $\mathrm{V}_{35 \mathrm{~Gy}}(\mathrm{~mL})$ & $110.4(77.9-253.4)$ & $104.8(74.5-160.5)$ & 0.5566 \\
\hline $\mathrm{V}_{45 G \mathrm{y}}(\mathrm{mL})$ & $83.0(50.1-176.8)$ & $31.5(26.1-48.2)$ & 0.0371 \\
\hline \multicolumn{4}{|l|}{ Large intestine } \\
\hline $\mathrm{V}_{30 \mathrm{~Gy}}(\mathrm{~mL})$ & \multicolumn{2}{|c|}{$142.7(110.8-271.7)$} & 0.0137 \\
\hline $\mathrm{V}_{35 \mathrm{~Gy}}(\mathrm{~mL})$ & $106.4(79.5-188.2)$ & $102.7(65.6-132.6)$ & 0.1602 \\
\hline $\mathrm{V}_{45 G \mathrm{y}}(\mathrm{mL})$ & $61.3(54.3-121.5)$ & $29.4(19.7-38.2)$ & 0.0273 \\
\hline \multicolumn{4}{|l|}{ Femoral head } \\
\hline Volume (mL) & \multicolumn{2}{|c|}{$104.1(83.7-111.3)$} & \\
\hline$V_{30 G y}(\%)$ & $49.2(39.8-58.9)$ & $52.7(46.5-64.9)$ & 0.4316 \\
\hline$V_{40 G y}(\%)$ & $13.9(8.5-18.2)$ & $15.4(9.9-19.5)$ & 0.8457 \\
\hline $\mathrm{V}_{44 G \mathrm{y}}(\%)$ & $10.8(5.24-14.6)$ & $3.7(1.9-8.1)$ & 0.1055 \\
\hline \multicolumn{4}{|l|}{ Pelvic bone } \\
\hline Volume (mL) & \multicolumn{2}{|c|}{851.2 (809.1-934.8) } & \\
\hline Mean dose (Gy) & $25.6(24.1-27.2)$ & $31.4(30.1-31.8)$ & 0.002 \\
\hline $\mathrm{V}_{10 \mathrm{~Gy}}(\mathrm{~mL})$ & $609.5(555.1-662.4)$ & $772.2(689.6-836.4)$ & 0.002 \\
\hline $\mathrm{V}_{20 \mathrm{~Gy}}(\mathrm{~mL})$ & $559.1(510.7-605.4)$ & $680.4(603.6-716.7)$ & 0.002 \\
\hline $\mathrm{V}_{30 G y}(\mathrm{~mL})$ & $449.6(413.6-496.3)$ & $560.3(488.3-573.8)$ & 0.0137 \\
\hline $\mathrm{V}_{40 \mathrm{~Gy}}(\mathrm{~mL})$ & $194.6(168.7-223.5)$ & $336.2(307.2-357.0)$ & 0.0039 \\
\hline $\mathrm{V}_{50 \mathrm{~Gy}}(\mathrm{~mL})$ & 89.9 (70.2-101.8) & $27.7(8.7-40.6)$ & 0.002 \\
\hline
\end{tabular}

Values are presented as median (interquartile range).

3DCRT, three-dimensional conformal radiotherapy; IMRT, intensity-modulated radiotherapy; PTV54, planning target volume receiving 54 Gy; PTV45, planning target volume receiving $45 \mathrm{~Gy} ; D_{X \% \text {; }}$ the dose covering $X \%$ of the volume of the structure; $V_{X G y}(\%)$, the percentage of the structure volume receiving $X G y ; V_{X G Y}(m L)$, the volume of the structure receiving $X G y$.

a) The reference dose was $54 \mathrm{~Gy}$.

irradiated left ipsilateral metastatic inguinal lymph node, 13 months after the initial day of chemoradiotherapy (Fig. 4). She received the salvage local electron radiotherapy and adjuvant chemotherapy. The 3-year LRC, PFS, and OS were 88.9\% (95\% confidence interval, 43.3\%-98.4\%), 80.0\% (95\% confidence interval, 40.9\%-94.6\%) (Fig. 5), and 100\%, respectively.

\section{Late toxicity}

No patient developed any $\geq$ grade 3 late adverse event of the skin, gastrointestinal tract, or urogenital system. Anal sphincter tone was well-preserved and no anal canal stricture was noted in any patient. All patients could control flatus and fecal continence as well as can normal individuals. 

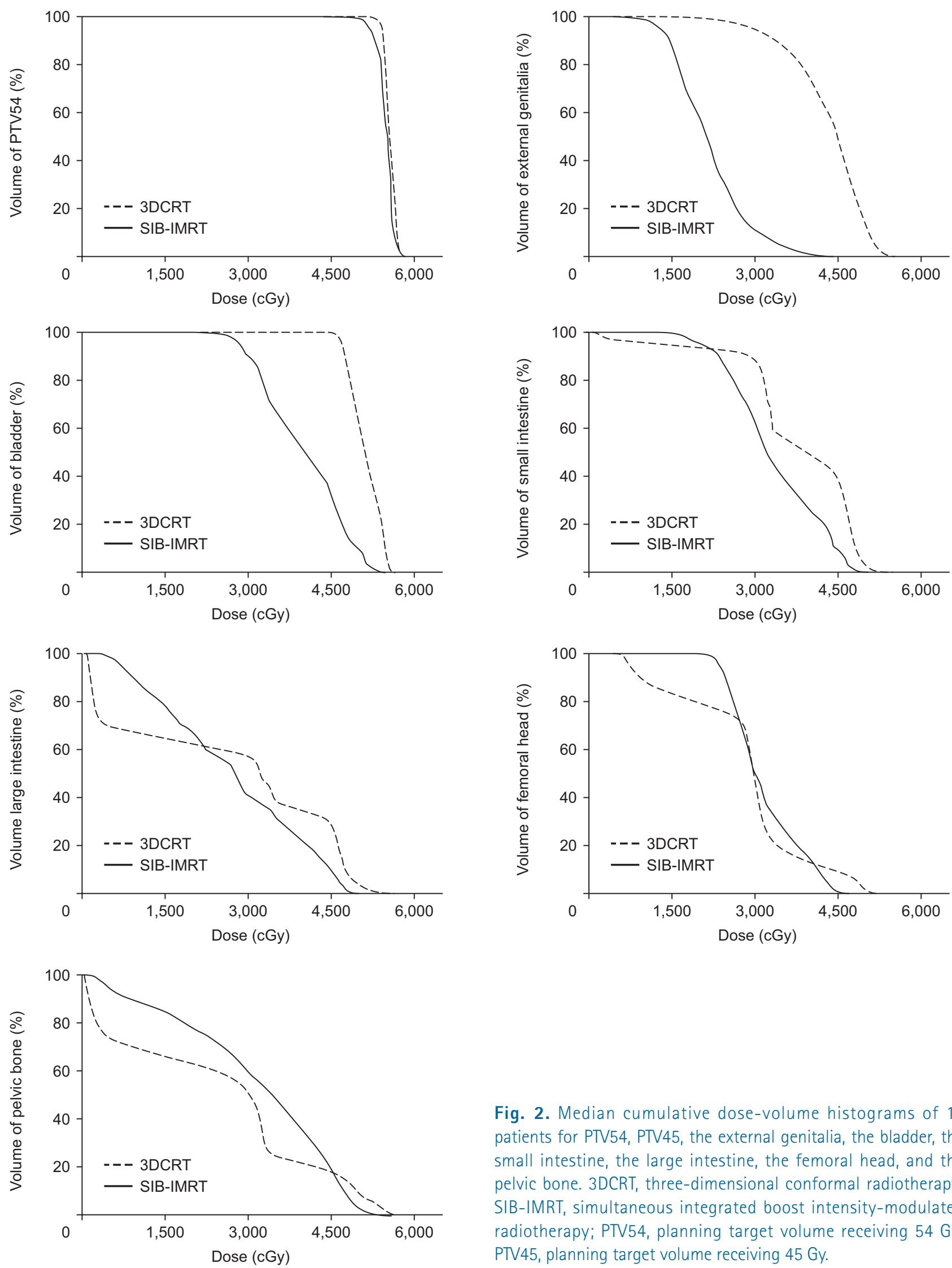

Fig. 2. Median cumulative dose-volume histograms of 10 patients for PTV54, PTV45, the external genitalia, the bladder, the small intestine, the large intestine, the femoral head, and the pelvic bone. 3DCRT, three-dimensional conformal radiotherapy; SIB-IMRT, simultaneous integrated boost intensity-modulated radiotherapy; PTV54, planning target volume receiving $54 \mathrm{~Gy}$; PTV45, planning target volume receiving $45 \mathrm{~Gy}$. 
Table 3. Acute adverse events

\begin{tabular}{|c|c|c|c|}
\hline & Grade $1 / 2 / 3 / 4$ & $\geq$ Grade $3(\%)$ & Grade $4(\%)$ \\
\hline Hematological & $1 / 0 / 4 / 5$ & - & 50 \\
\hline Leukocytopenia & $1 / 0 / 6 / 3$ & - & 30 \\
\hline Neutropenia & $0 / 1 / 4 / 4$ & - & 40 \\
\hline Anemia & $2 / 6 / 0 / 0$ & - & 0 \\
\hline Thrombocytopenia & $3 / 1 / 2 / 2$ & - & 20 \\
\hline Non-hematological & $0 / 7 / 3 / 0$ & 30 & - \\
\hline Fatigue & $8 / 1 / 0 / 0$ & 0 & - \\
\hline Nausea/appetite loss & $5 / 4 / 0 / 0$ & 0 & - \\
\hline Diarrhea & $5 / 4 / 1 / 0$ & 10 & - \\
\hline Dysuria/urinary frequency & $4 / 3 / 0 / 0$ & 0 & - \\
\hline Perineal dermatitis & $2 / 6 / 2 / 0$ & 20 & - \\
\hline Anal pain & $1 / 7 / 2 / 0$ & 20 & - \\
\hline
\end{tabular}
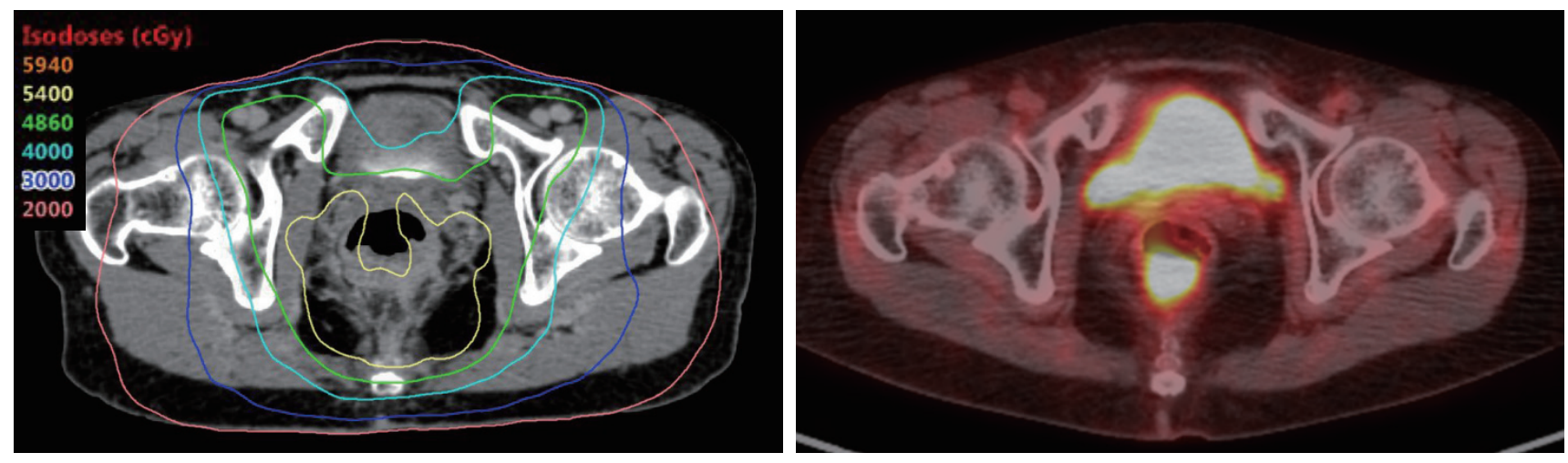

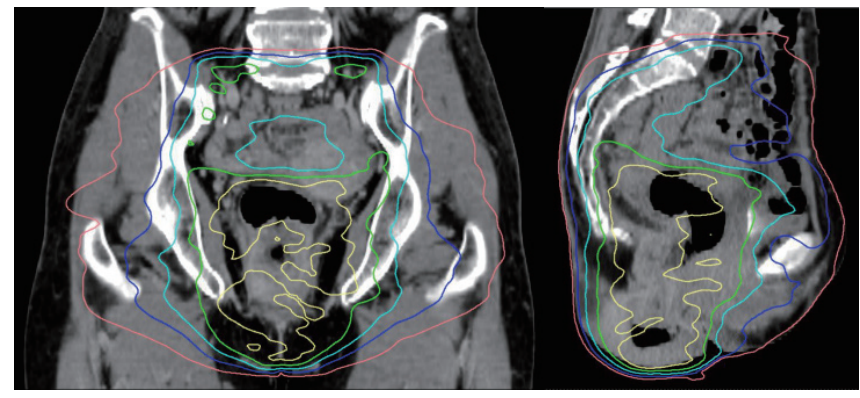

A

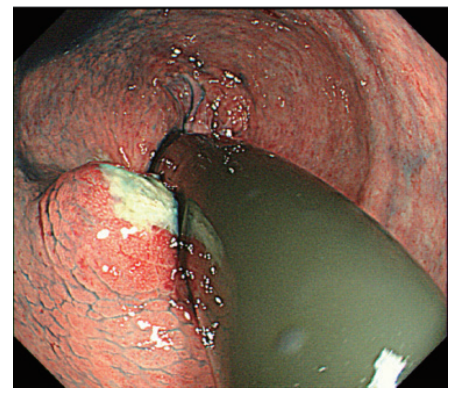

B

Fig. 3. Recurrence site after chemoradiotherapy using simultaneous integrated boost intensity-modulated radiotherapy (SIB-IMRT) for the patient with cT3N3M0 anal squamous cell carcinoma. (A) Axial, coronal, and sagittal planes with isodose distribution of SIB-IMRT. (B) Intraluminal recurrence image of 18F-fluorodeoxyglucose positron emission tomography/computed tomography and endoscopic images 32 months after chemoradiotherapy using SIB-IMRT.

\section{Discussion and Conclusion}

The planning study showed that SIB-IMRT spared the external genitalia, the bladder, and the intestine but allowed the planned dose delivery to the target. These dosimetric advantages of SIB-IMRT reduced the numbers of nonhematological acute and late toxicities but afforded good locoregional control (Table 4).

Conventional radiotherapy technique such as 3DCRT was the established radiotherapy technique in the chemoradiotherapy for ASCC. The NCCN guideline 2017 version 2 cited the extent of fields and doses of 3DCRT for ASCC based on the RTOG 9811 protocol. Thus, the extent of fields and doses of RTOG 9811 is one of the standard irradiation fields for 3DCRT for ASCC. The 

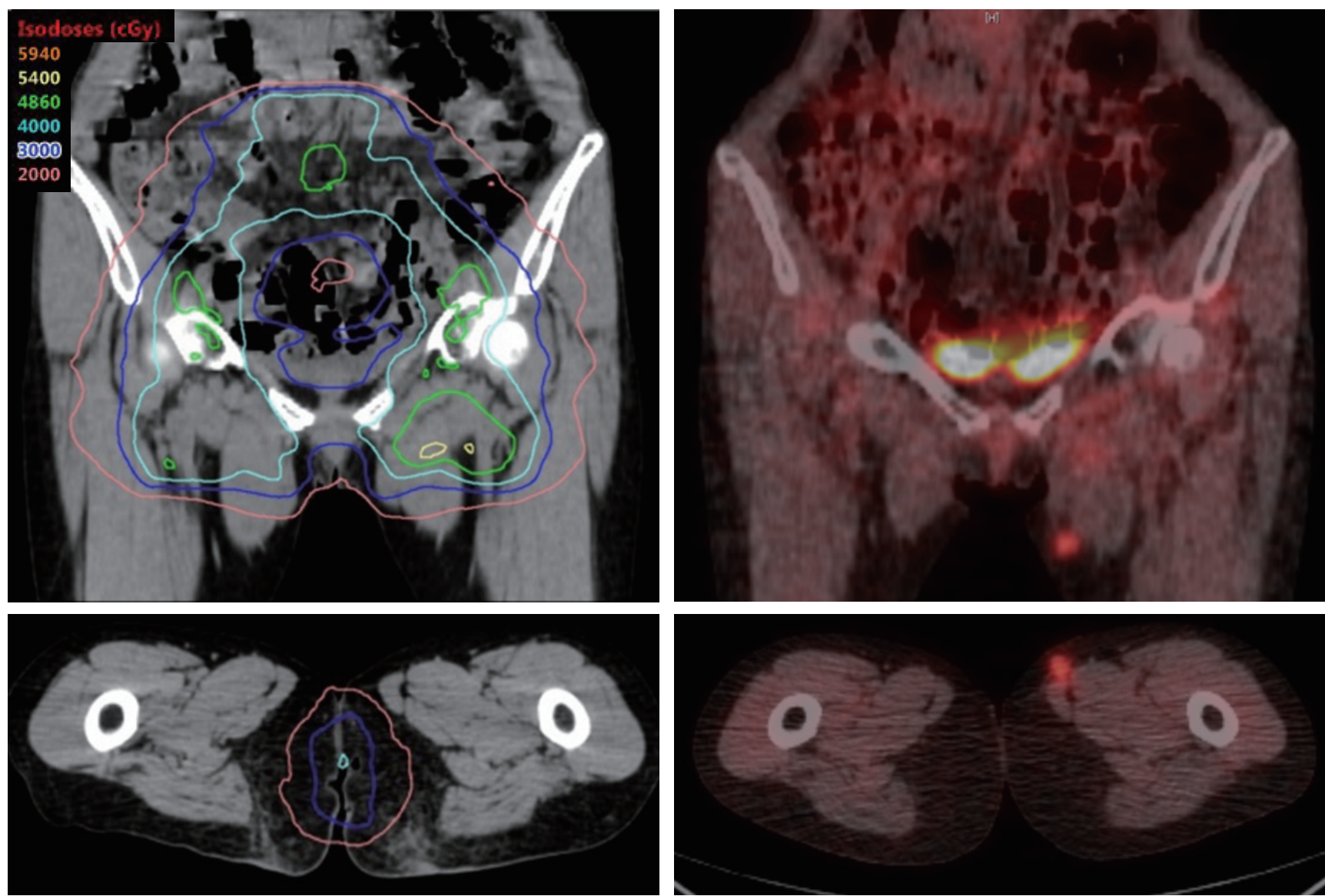

A

B

Fig. 4. Recurrence site after chemoradiotherapy using simultaneous integrated boost intensity-modulated radiotherapy (SIB-IMRT) for the patient with cT2N2M1 anal squamous cell carcinoma (ASCC). (A) Axial and coronal planes with isodose distribution for cT2N2M1 ASCC. (B) Femoral lymph nodal recurrence image of computed tomography.

SIB-IMRT was now becoming popular radiotherapy technique for ASCC. Multi-institutional phase II study (RTOG 0529) adopted the SIB-IMRT in the chemoradiotherapy for ASCC, which suggested the reduction of acute toxicity compared with 3DCRT following RTOG 9811. The extent of elective nodal region differed between them. 3DCRT field was small to reduce the doses to intestine than that of SIB-IMRT. Thus, the simple dosimetric comparison between 3DCRT and SIB-IMRT for ASCC includes the problem. We considered that 3DCRT following RTOG 9811 for the larger elective nodal region and doses adopted by SIB-IMRT plans following RTOG 0529 wasn't clinically validated, and its feasibility wasn't unknown. Thus, the current study avoided the comparison of the doses to PTV45 between 3DCRT and SIB-IMRT.

IMRT spares organs at risk, but target coverage may be compromised. The dose distribution and target coverage of PTV54 were significantly less homogenous and poorer on SIB-
IMRT than 3DCRT in this study. These are drawbacks of SIBIMRT; however, the absolute differences in dose indices for target coverage were small. The median $\mathrm{D}_{2 \%}$ and $\mathrm{D}_{98 \%}$ differed by about 0.5 and $2.7 \mathrm{~Gy}$ of the $54 \mathrm{~Gy}$, respectively. Such differences were unlikely to compromise locoregional control by SIB-IMRT. Indeed, we found that dosimetric advantages of SIB-IMRT were associated with reducing toxicity, high complete response and LRC. The significant clinical benefit was afforded by SIB-IMRT to reduce the number of acute nonhematological events without compromising effectiveness.

A multi-institutional phase II study of chemoradiotherapy using SIB-IMRT reported the number of days on which radiotherapy had to be interrupted were fewer in number than the number associated with historical control 3DCRT Treatment breaks were required by $49 \%$ of SIB-IMRT [5] compared to $62 \%$ of 3 DCRT [1] patients, and the median duration of treatment interruption was 0 days (range, 0 to 


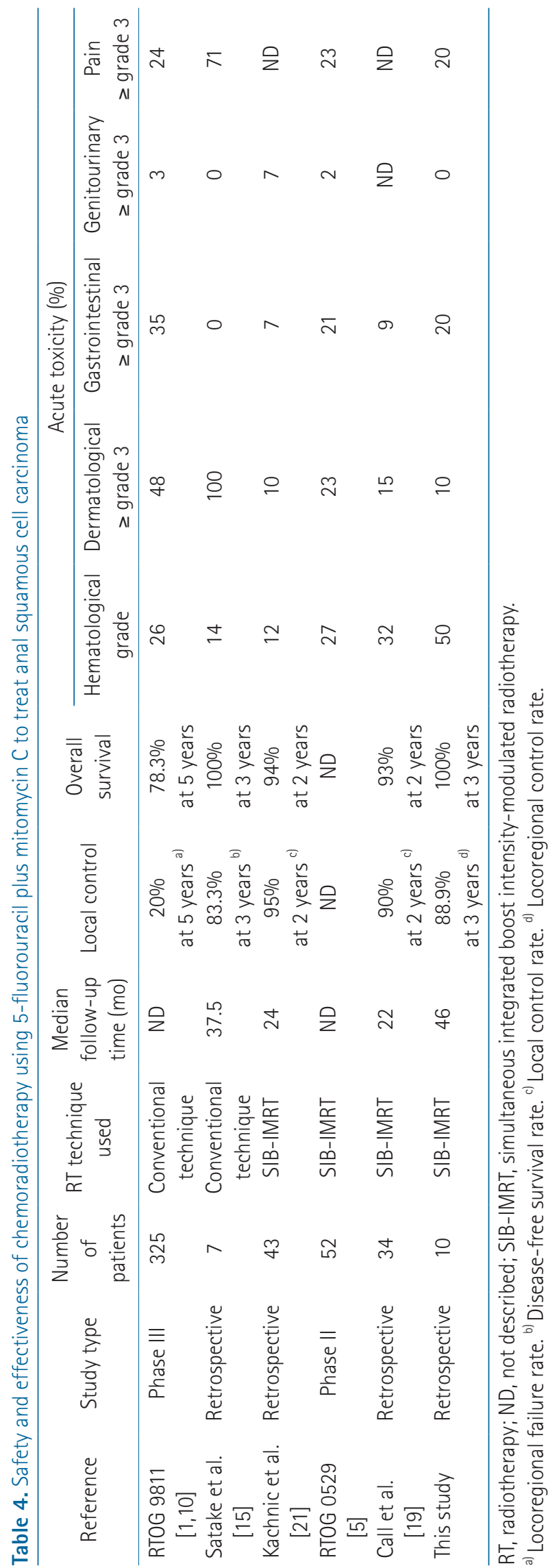

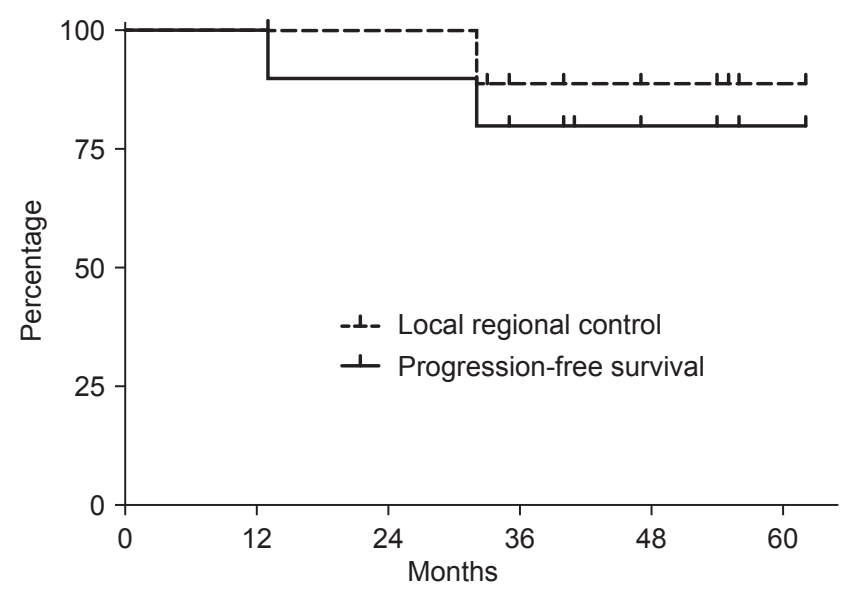

Fig. 5. Local regional control and progression free-survival survival.

12 days) in SIB-IMRT patients [5] compared to 3 days (range, 0 to 33 days) in those undergoing 3DCRT [1]. In the current study, SIB-IMRT reduced the dose to intestine and external genitalia and alleviated acute toxicities compared with 3DCRT (RTOG 9811 [1] or ACT II [2]), thus possibly avoiding unplanned prolongation of the overall treatment time. This would also increase treatment effectiveness. The overall treatment time was a significant prognostic factor in multi-institutional phase III trial of chemoradiotherapy using conventional radiotherapy for ASCC [10]. Thus, reduction in the number of acute toxicities during SIB-IMRT is probably beneficial to treatment effectiveness.

The SIB-IMRT dose to the pelvic bone was higher than that of 3DCRT. The principal reason was the difference in the extent of the elective nodal irradiation between SIB-IMRT following RTOG 0529 and 3DCRT following RTOG 9811. Forty-five Gy was prescribed to the mesorectum, presacrum, and bilateral obturator; internal and external iliac; and inguinal nodes in SIB-IMRT plans (RTOG 0529 and the current study) but cranial portion of pelvic lymph node was spared after 30.6 Gy in 3DCRT plans (RTOG 9811 [1] or ACT II [2]). The elective nodal region for ASCC lies along the pelvic bone. Two retrospective studies have suggested that dosing of the pelvic bone was associated with acute hematological toxicity $[11,12]$. The increased doses to the pelvic bone in SIB-IMRT possibly caused the higher incidence of hematological toxicity in the current study, although the racial difference also probably affected $[1,2]$. Thus, we need to remind that the extent of elective nodal irradiation differed between RTOG 9811 and RTOG 0529, when we apply SIB-IMRT to the patients with ASCC following the RTOG 0529 protocol. Then, the dose to the pelvic bone should 
be recognized as a cause of hematologic adverse events in chemoradiotherapy for ASCC.

The incidence of late adverse events was rather high after chemoradiotherapy using 3DCRT for ASCC. A multiinstitutional phase III trial employing conventional radiotherapy found that late adverse events in subcutaneous tissue, the intestine, and bladder of $\geq$ grade 3 occurred in $11 \%$ of patients during a median follow-up period of 2.51 years [13]. Usefully, IMRT reduces the volume of normal tissue receiving high doses, possibly alleviating late toxicities. Indeed, no patient experienced a late toxicity of $\geq$ grade 3 in this study. Conformal dose delivery during SIB-IMRT significantly reduced the doses to the external genitalia, intestine, and bladder, compared to 3DCRT, which likely reduced the late toxicities associated with chemoradiotherapy for ASCC. The current results will support the use of IMRT for ASCC.

Our study had the limitation that we had few patients. This is because ASCC is rare in Asia $[14,15]$. To the best of our knowledge, all available information on IMRT for ASCC has been collected in the West [5,16-22]. Such data may apply to Asians; however, Westerners have larger pelvic cavities and more abdominal fat than Asians. Such anatomical differences may render it difficult to retain target coverage while sparing organs at risk in Asians. This study suggested that it was feasible to use chemoradiotherapy (SIB-IMRT with 5-fluorouracil and mitomycin C) for ASCC even in Asians for whom detailed information on the dose/volume metrics of the targets and organs at risk was available (Table 2). Our findings will help radiation oncologists to create SIB-IMRT plans, and physicians to understand the utility of SIB-IMRT for ASCC. We hope that our work will promote the use of SIB-IMRT for ASCC in clinical practice.

SIB-IMRT for ASCC delivered the planned doses to gross tumors and elective nodal region but significantly reduced the doses to the external genitalia, bladder, and intestine, compared to 3DCRT. These dosimetric advantages of SIBIMRT reduced the numbers of acute and late dermatological, genitourinary, and intestinal toxicities, without compromising locoregional control, in ASCC patients who underwent chemoradiotherapy using 5 -fluorouracil and mitomycin $\mathrm{C}$. The information of dose volume indices and clinical outcome of this study will be useful to create the protocol in the future prospective study.

\section{Conflict of Interest}

No potential conflict of interest relevant to this article was reported.

\section{Acknowledgments}

This research was partially supported by grants from a nonprofit organization (Tokyo Cooperative Oncology Group).

\section{References}

1. Ajani JA, Winter KA, Gunderson LL, et al. Fluorouracil, mitomycin, and radiotherapy vs fluorouracil, cisplatin, and radiotherapy for carcinoma of the anal canal: a randomized controlled trial. JAMA 2008;299:1914-21.

2. James RD, Glynne-Jones R, Meadows HM, et al. Mitomycin or cisplatin chemoradiation with or without maintenance chemotherapy for treatment of squamous-cell carcinoma of the anus (ACT II): a randomised, phase 3, open-label, $2 \times 2$ factorial trial. Lancet Oncol 2013;14:516-24.

3. Chen YJ, Liu A, Tsai PT, et al. Organ sparing by conformal avoidance intensity-modulated radiation therapy for anal cancer: dosimetric evaluation of coverage of pelvis and inguinal/femoral nodes. Int J Radiat Oncol Biol Phys 2005;63:274-81.

4. Brooks CJ, Lee YK, Aitken K, Hansen VN, Tait DM, Hawkins MA. Organ-sparing Intensity-modulated radiotherapy for anal cancer using the ACTII schedule: a comparison of conventional and intensity-modulated radiotherapy plans. Clin Oncol (R Coll Radiol) 2013;25:155-61.

5. Kachnic LA, Winter K, Myerson RJ, et al. RTOG 0529: a phase 2 evaluation of dose-painted intensity modulated radiation therapy in combination with 5-fluorouracil and mitomycin-C for the reduction of acute morbidity in carcinoma of the anal canal. Int J Radiat Oncol Biol Phys 2013;86:27-33.

6. Clivio A, Fogliata A, Franzetti-Pellanda A, et al. Volumetricmodulated arc radiotherapy for carcinomas of the anal canal: a treatment planning comparison with fixed field IMRT. Radiother Oncol 2009;92:118-24.

7. Vieillot S, Azria D, Lemanski C, et al. Plan comparison of volumetric-modulated arc therapy (RapidArc) and conventional intensity-modulated radiation therapy (IMRT) in anal canal cancer. Radiat Oncol 2010;5:92.

8. Ugurluer G, Ballerini G, Moeckli R, Matzinger O, Bourhis J, Ozsahin M. Helical tomotherapy for the treatment of anal canal cancer: a dosimetric comparison with 3D conformal radiotherapy. Tumori 2015;101:268-72.

9. Myerson RJ, Garofalo MC, El Naqa I, et al. Elective clinical target volumes for conformal therapy in anorectal cancer: a radiation therapy oncology group consensus panel contouring atlas. Int J Radiat Oncol Biol Phys 2009;74:824-30. 
10. Ben-Josef E, Moughan J, Ajani JA, et al. Impact of overall treatment time on survival and local control in patients with anal cancer: a pooled data analysis of Radiation Therapy Oncology Group trials 87-04 and 98-11. J Clin Oncol 2010;28:5061-6.

11. Bazan JG, Luxton G, Mok EC, Koong AC, Chang DT. Normal tissue complication probability modeling of acute hematologic toxicity in patients treated with intensity-modulated radiation therapy for squamous cell carcinoma of the anal canal. Int J Radiat Oncol Biol Phys 2012;84:700-6.

12. Mell LK, Schomas DA, Salama JK, et al. Association between bone marrow dosimetric parameters and acute hematologic toxicity in anal cancer patients treated with concurrent chemotherapy and intensity-modulated radiotherapy. Int J Radiat Oncol Biol Phys 2008;70:1431-7.

13. Gunderson LL, Winter KA, Ajani JA, et al. Long-term update of US GI intergroup RTOG 98-11 phase III trial for anal carcinoma: survival, relapse, and colostomy failure with concurrent chemoradiation involving fluorouracil/mitomycin versus fluorouracil/cisplatin. J Clin Oncol 2012;30:4344-51.

14. Takashima A, Shimada Y, Hamaguchi T, et al. Current therapeutic strategies for anal squamous cell carcinoma in Japan. Int J Clin Oncol 2009;14:416-20.

15. Satake H, Yoshino T, Sasaki T, et al. Early clinical outcomes of anal squamous cell carcinoma treated with concurrent chemoradiotherapy with 5 -Fluorouracil plus mitomycin $\mathrm{C}$ in Japanese patients: experience at a single institution. Jpn J Clin Oncol 2012;42:861-4.
16. Joseph $K$, Nijjar $Y$, Warkentin $H$, et al. Prospective phase II study of tomotherapy based chemoradiation treatment for locally advanced anal cancer. Radiother Oncol 2015;117:234-9.

17. Milano MT, Jani AB, Farrey KJ, Rash C, Heimann R, Chmura SJ. Intensity-modulated radiation therapy (IMRT) in the treatment of anal cancer: toxicity and clinical outcome. Int J Radiat Oncol Biol Phys 2005;63:354-61.

18. Dasgupta $T$, Rothenstein D, Chou JF, et al. Intensity-modulated radiotherapy vs. conventional radiotherapy in the treatment of anal squamous cell carcinoma: a propensity score analysis. Radiother Oncol 2013;107:189-94.

19. Call JA, Haddock MG, Quevedo JF, Larson DW, Miller RC Concurrent chemotherapy and intensity modulated radiation therapy in the treatment of anal cancer: a retrospective review from a large academic center. Pract Radiat Oncol 2013;3:2631.

20. Pepek JM, Willett CG, Wu OJ, Yoo S, Clough RW, Czito BG. Intensity-modulated radiation therapy for anal malignancies: a preliminary toxicity and disease outcomes analysis. Int J Radiat Oncol Biol Phys 2010;78:1413-9.

21. Kachnic LA, Tsai HK, Coen JJ, et al. Dose-painted intensitymodulated radiation therapy for anal cancer: a multiinstitutional report of acute toxicity and response to therapy. Int J Radiat Oncol Biol Phys 2012;82:153-8.

22. Hodges JC, Beg MS, Das P, Meyer J. Cost-effectiveness analysis of intensity modulated radiation therapy versus 3-dimensional conformal radiation therapy for anal cancer. Int J Radiat Oncol Biol Phys 2014;89:773-83. 